\title{
Coherent-Radiation Spectroscopy of Few-Femtosecond Electron Bunches Using a Middle-Infrared Prism Spectrometer
}

\author{
T. J. Maxwell, ${ }^{1, *}$ C. Behrens, ${ }^{1,2}$ Y. Ding, ${ }^{1}$ A. S. Fisher, ${ }^{1}$ J. Frisch, ${ }^{1}$ Z. Huang, ${ }^{1}$ and H. Loos ${ }^{1}$ \\ ${ }^{1}$ SLAC National Accelerator Laboratory, Menlo Park, California 94025, USA \\ ${ }^{2}$ Deutsches Elektronen-Synchrotron DESY, Notkestraße 85, 22607 Hamburg, Germany
}

(Received 2 August 2013; published 28 October 2013)

\begin{abstract}
Modern, high-brightness electron beams such as those from plasma wakefield accelerators and free-electron laser linacs continue the drive to ever-shorter bunch durations. In low-charge operation $(\sim 20 \mathrm{pC})$, bunches shorter than $10 \mathrm{fs}$ are reported at the Linac Coherent Light Source (LCLS). Though suffering from a loss of phase information, spectral diagnostics remain appealing as compact, low-cost bunch duration monitors suitable for deployment in beam dynamics studies and operations instrumentation. Progress in middle-infrared (MIR) imaging has led to the development of a single-shot, MIR prism spectrometer to characterize the corresponding LCLS coherent beam radiation power spectrum for fewfemtosecond scale bunch length monitoring. In this Letter, we report on the spectrometer installation as well as the temporal reconstruction of 3 to 60 fs-long LCLS electron bunch profiles using single-shot coherent transition radiation spectra.
\end{abstract}

DOI: 10.1103/PhysRevLett.111.184801

PACS numbers: 29.27.- a, 41.60.Dk, 41.75.Ht

The generation and characterization of femtosecond duration electron bunches are of paramount importance in high-energy collider, plasma wakefield accelerator (PWFA), and x-ray free-electron laser (FEL) applications. In a PWFA, the duration of the accelerated bunch is intrinsically shorter than the plasma oscillation period which is typically on the order of 100 fs [1]. In x-ray FELs, reducing the charge per bunch can alleviate internal space charge and other nonlinear, beam-induced forces allowing for a corresponding reduction of the minimum possible duration. For example, with tens of picocoloumb bunch charges, the Linac Coherent Light Source (LCLS) [2] is capable of generating electron and x-ray pulses with durations of just a few femtoseconds [3]. Diagnosis of the longitudinal electron distribution is, therefore, highly desirable for FEL optimization studies and presents a unique challenge to the resolution of existing diagnostics, reaching into the few-fs scale. In this Letter, we present first results using a newly developed frequency-domain diagnostic with advantages of economy and robustness over direct time-domain diagnostics [4-6] for the LCLS. The prism-based spectrometer demonstrated yields novel, single-shot measurements over a wide, middle-infrared (MIR) spectral range previously unexplored in longitudinal beam diagnostics.

Frequency-domain bunch length measurements are based on observing the power spectrum of light from an electron bunch undergoing a radiative process, occurring when the beam experiences a change in trajectory or medium $[7,8]$. In the low-frequency range, where $\kappa \lesssim$ $\left(2 \pi \sigma_{z}\right)^{-1}$ with $\sigma_{z}$ the electron bunch length and $\kappa$ the spatial frequency $\kappa \equiv 1 / \lambda$, the radiated power has a wellknown coherent enhancement that scales with the number of electrons $N$ over the incoherent beam radiation background. In the one-dimensional bunch limit, where the transverse electron beam size is much smaller than the transverse coherence length, the coherent spectral density profile can be expressed as

$$
I\left(\boldsymbol{r}_{\perp}, \kappa\right) \approx N^{2} I_{e}\left(\boldsymbol{r}_{\perp}, \kappa\right)|f(\kappa)|^{2},
$$

where the form factor $f(\kappa)$ is defined as the Fourier transform of the unit-normalized longitudinal charge distribution $\rho(z)$ as

$$
f(\kappa)=\int_{-\infty}^{\infty} \rho(z) \exp (2 \pi i \kappa z) d z .
$$

The function $I_{e}$ is the spatial distribution in transverse coordinate $\boldsymbol{r}_{\perp}$ of spectral component $\boldsymbol{\kappa}$ for an individual electron. The form of $I_{e}\left(\boldsymbol{r}_{\perp}, \kappa\right)$ varies depending on the radiative process and imaging optics used. Coherent transition radiation (CTR) was used for this Letter, produced when the beam strikes a thin foil. Descriptions of $I_{e}$ for CTR have been rigorously studied [8-12] with the transverse coherence condition on the beam size $\sigma_{r} \ll \gamma \lambda / 2 \pi$ for a beam with a Lorentz factor $\gamma$. Equation (1) is the basis of CTR spectroscopy as a bunch length diagnostic. The spectrum from the far infrared $(\kappa \rightarrow 0)$ through $\kappa \approx$ $\left(2 \pi \sigma_{z}\right)^{-1}$ is measured. With $\sigma_{z}$ inversely proportional to the measured spectral bandwidth, the increasingly shorter bunches from high-brightness beams demand ever-higher bandwidth spectrometry.

In the LCLS beam line, shown in Fig. 1, linac sections ( $L 0, L 1, L 2$, and $L 3$ ) bring the beam to a final energy of 3.5-14 GeV prior to reaching the undulator section where ultrashort x-ray pulses are generated. The longitudinal dispersion of bunch compressor chicanes $\mathrm{BC} 1$ and $\mathrm{BC} 2$ reduce the length of the linearly energy chirped electron bunch. Existing LCLS relative bunch length monitor 


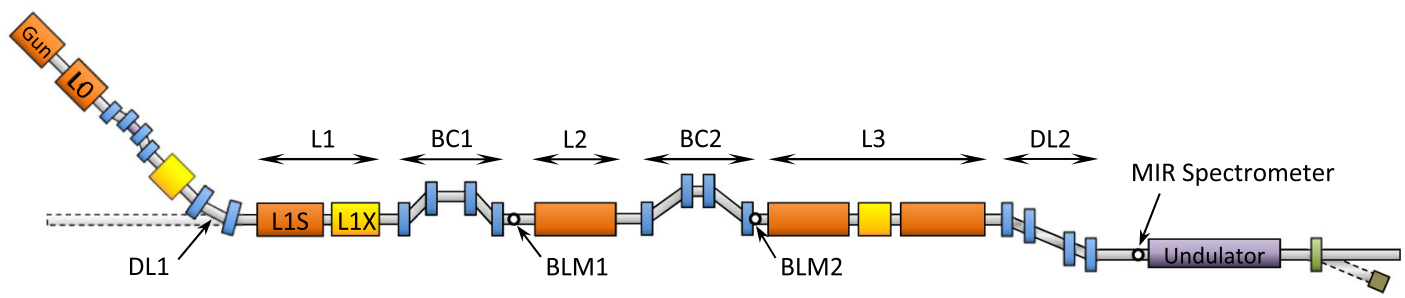

FIG. 1 (color online). Layout of the LCLS beam line with locations of relevant diagnostics along the LCLS linac (see text).

(BLM) locations are also shown $[12,13]$. These record the integrated coherent edge radiation power to provide a relative measure of bunch length. Absolute measurement is achieved by calibrating BLM detector levels at welldefined beam conditions to LCLS transverse deflecting mode cavity measurements.

The minimum achievable $\sigma_{z}$ is expected to be $<10 \mu \mathrm{m}$ at $150 \mathrm{pC}$, scaling to just $1 \mu \mathrm{m}$ at $20 \mathrm{pC}$ [3]. As $\sigma_{z}$ drives the $\mathrm{x}$-ray pulse length generated in the undulator, absolute measurement just prior to the undulator entrance is desired. Small changes to $\sigma_{z}$ imparted after $\mathrm{BC} 2$ from beaminduced effects (e.g., space charge and wakefields) combined with the weak dispersion of the dogleg DL2 (Fig. 1) can then be observed.

Single-shot spectroscopy of the MIR coherent beam radiation spanning $\lambda=1-40 \mu \mathrm{m}$ is, therefore, needed to absolutely determine $\sigma_{z}$ over the range of achievable bunch lengths. Many instruments have evolved to characterize CTR for longer wavelengths and were considered for extension down to the few- $\mu \mathrm{m}$ bunch regime [14-21].

To cover the broad MIR range, we have combined the bandwidth of the cascaded grating configuration [21] with the reduced complexity of the single-grating polychromator $[19,20]$ by using a conventional prism spectrometer design. The weaker, harmonic-independent prism dispersion makes wide bandwidth coverage straightforward without the need for order sorting. Tradeoffs for choosing a prism over gratings are lower spectral resolution and the dispersion nonlinearity introduced by the prism material properties.

Details on the MIR spectrometer design have been previously discussed [22] with the final system described in Fig. 2. The optics are enclosed and purged to $<0.1 \%$ relative humidity with dried air to minimize atmospheric water vapor absorption bands. The $10^{\circ}$ apex-angle prism $(H)$ was fabricated from thallium bromoiodide (KRS-5) owing to its flat transmission and strong dispersion over the $\lambda=$ $0.8-40 \mu \mathrm{m}$ range. Reflective optics are used otherwise and chosen to provide diffraction-limited imaging at the linear detector array $(J)$ which consists of 128 lead zirconate titanate pyroelectric elements with $100 \mu \mathrm{m}$ spacing.

Calibration is complicated by the relative scarcity of bright MIR sources. Wavelength calibration was performed using an $18 \mathrm{~W}, 1200^{\circ} \mathrm{C}$ silicon nitride blackbody source. A series of bandpass filters were used to generate spectral lines. The radiator provided measurable illumination over the range $\lambda=1.6-16 \mu \mathrm{m}$ after chopping, collimation, and filtering.

For interpolation and extrapolation of the position $x$ on the array for a given spectral component $\kappa$, a curve was fit to the ideal function $x_{\text {calc }}(\kappa)$ calculated from Snell's law for the KRS-5 index of refraction [23] with additional slope and offset parameters allowed for small detector misalignment and an arbitrary spatial offset of the array. The FWHM spectral resolution $\Delta \kappa$ is computed from the spatial dispersion $d x / d \kappa$ as $\Delta \kappa \approx(d x / d \kappa)^{-1} \Delta x$, where $\Delta x$ is the diffraction-limited image of the slit at the detector. Results are shown in Fig. 3.

Accurate bunch length determination is predicated on characterizing the total spectral amplitude transfer function $T(\kappa)$ for the system. Where the transverse extent of spectral components can be neglected, the measured spatial power density $I_{\text {meas }}[x(\kappa)]$ along coordinate $x$ at the array is approximately related to the term $|f(\kappa)|^{2}$ via several contributing factors as

$$
\begin{aligned}
I_{\text {meas }}[x(\kappa)] & =(d x / d \kappa)^{-1} T_{\text {det }}(\kappa) T_{\text {abs }}(\kappa)\left[N^{2} I_{e}(\kappa)|f(\kappa)|^{2}\right] \\
& =T(k)|f(\kappa)|^{2} .
\end{aligned}
$$

The $d x / d \kappa$ term represents the nonlinear mapping of the spectral density to the measured spatial density via the dispersion. Other terms are the detector response $T_{\text {det }}$

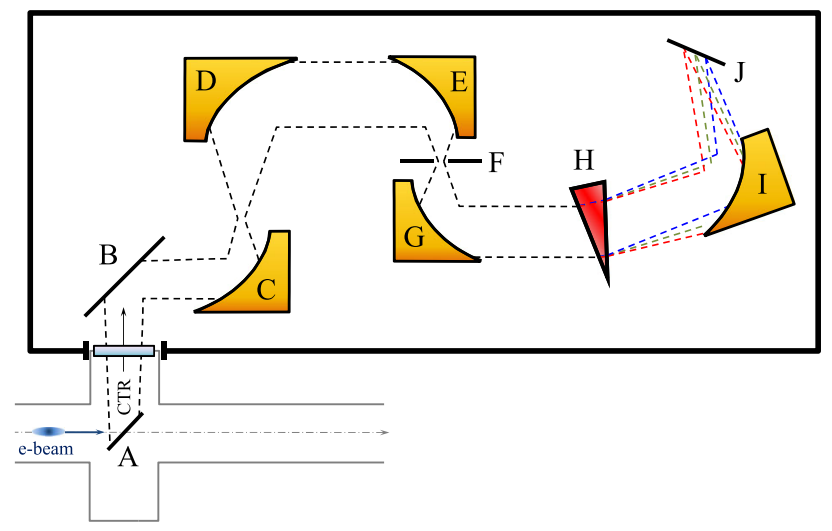

FIG. 2 (color online). Schematic for MIR spectroscopy of CTR with CTR envelope shown as dashed lines. CTR from a thin foil inserted into the electron beam $(A)$ is imaged with reflective optics $(B-E)$ to the spectrometer entrance slit $(F)$, chromatically dispersed by a custom KRS-5 prism $(H)$, then spectrally resolved on a linear detector array $(J)$. 

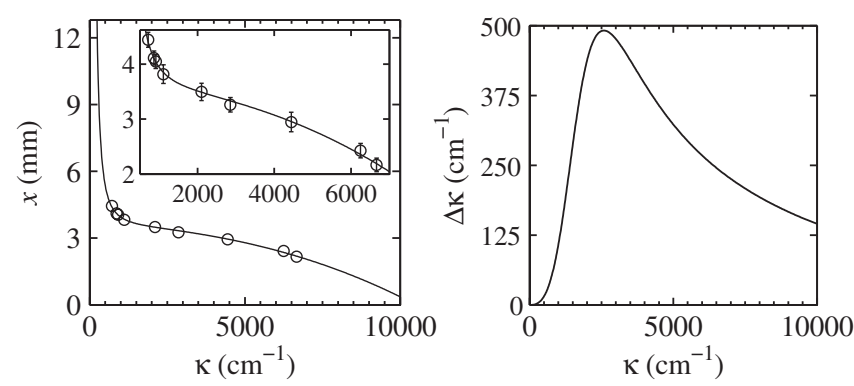

FIG. 3. Fit of computed detector position $x$ as a function of spatial frequency $\kappa$ to a sample of spectral lines (left) with detail view over sampled points (left, inset) and spectral resolution $\Delta \kappa$ (right).

$[16,21]$, the spatially integrated single electron imaging contribution $I_{e}(\kappa)$ from Eq. (1), and $T_{\mathrm{abs}}$ from absorption by the prism and the vacuum and detector windows.

Data using the incoherent blackbody source lacked the resolution and range for accurate amplitude calibration. A procedure is being refined at the Advanced Light Source (ALS) MIR beam line to address this [24]. Where the beam being diagnosed is the only available coherent MIR source, an online approach was used to reconstruct $T(k)$ and is discussed here.

Assume the electron beam can be used to generate several MIR spectra by changing any independent accelerator parameter $\phi$ that will generate a different longitudinal distribution $\rho(z ; \phi)$. Then we can measure a $2 \mathrm{D}$ spectral intensity function

$$
I_{\text {meas }}(\kappa ; \phi)=T(\kappa)\left|f_{\text {meas }}(\kappa ; \phi)\right|^{2} \text {, }
$$

where the instrument response $T$ is independent of $\phi$ if $I_{e}$ is not significantly affected by $\phi$. If we can reasonably simulate the electron beam's dependence on $\phi$ to generate $\left|f_{\text {sim }}(\kappa ; \phi)\right|^{2}$, scale factors $T(\kappa)$ can be fit to satisfy Eq. (4). This tenuously assumes the spectra are sufficiently varied so that the $\phi$ and $\kappa$ dependencies can be separated.

A natural candidate for the independent parameter is the rf phase $\phi_{L 2}$ of accelerating section $L 2$ just prior to $\mathrm{BC} 2$ (Fig. 1). Changing $\phi_{L 2}$ changes the energy chirp put on the bunch prior to compression so that the resulting bunch length is varied. During this "chirp scan," the rf amplitude $V_{L 2}$ is adjusted so the energy gain $E_{L 2}=V_{L 2} \cos \phi_{L 2}$ is kept constant.

Scans were simulated using the LITRACK fast longitudinal phase space tracking and wakefield solver [25]. Unknown offsets to experimental settings are left free to improve fitting including the initial bunch energy spread, length and chirp, and the accelerator rf phases and amplitudes. Longitudinal profiles $\rho(z ; \phi)$ are simulated and Fourier transformed to $\left|f_{\text {sim }}(\kappa ; \phi)\right|^{2}$. A steepest-decent method is used to minimize the least-squares difference between simulated CTR spectra $\left|f_{\text {sim }}\right|^{2}$ and the corrected measured spectra $\left|f_{\text {meas }}\right|^{2}=I_{\text {meas }} / T$ with respect to simulation parameters, then minimized with respect to $T(\kappa)$ before the process repeats.

Chirp scan data for fitting were collected at LCLS with an electron beam energy of $13.4 \mathrm{GeV}$ and $40 \mathrm{pC}$ bunch charge. The scan was performed around maximum beam compression so the minimum expected bunch length is $\sim 2 \mu \mathrm{m}$, providing CTR across the full spectrometer range. Results are shown in Fig. 4 including $I_{\text {meas }}$, best candidates for $I_{\text {sim }}$ and $T$, and corrected data $\left|f_{\text {meas }}\right|^{2}$.

In $T(\kappa)$, a pile up is observed around $3000 \mathrm{~cm}^{-1}$ from the bottoming out of the $d x / d \kappa$ dispersion contribution [Eq. (3), Fig. 3]. Periodic minima every $2000 \mathrm{~cm}^{-1}$ in $\kappa$ (with additional structure) are also seen in parallel tests at the ALS MIR beam line (without structure), consistent with thin-film interference in the $1.5 \mu \mathrm{m}$-thick lead zirconate titanate layer of the detector array.

Minimum bunch length (maximum bandwidth) in Fig. 4 corresponds to the full-compression phase $\phi_{\text {full }}=-36^{\circ}$. A $\phi_{L 2}$-dependent spectral ringing structure appears in both simulation and the $T(\kappa)$-corrected data where $\left|\phi_{L 2}\right|<$ $\left|\phi_{\text {full }}\right|$ showing excellent agreement.

With $x(\kappa)$ and $T(\kappa)$ characterized, we would like to use measured spectral profiles to retrieve the longitudinal profiles $\rho(z)$. However, the spectral phase is unknown so the inverse Fourier transform to $\rho(z)$ cannot be uniquely determined. Common in CTR bunch length measurements $[16,17,21]$, the minimum Kramers-Kronig phase $\phi_{\min }$ is reconstructed following the prescription in [26-28]. This solution is still nonunique, and it is understood that ambiguities remain (cf. [28]). Several methods for low- $\kappa$ and high- $\kappa$ completion of measured spectra were analyzed where required. The choice of these was not found to significantly impact the results shown here.

Bunch length was again varied by changing the $L 2 \mathrm{rf}$ phase for the typical LCLS hard x-ray mode with a $13.4 \mathrm{GeV}$,
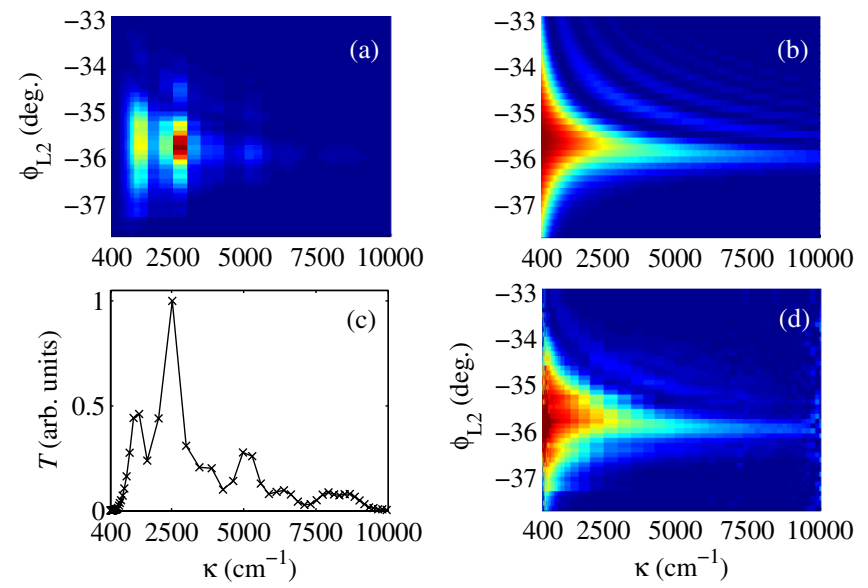

FIG. 4 (color online). $T(\kappa)$ reconstruction results for an $L 2$ chirp scan of a $40 \mathrm{pC}$ bunch with (a) raw data $I_{\text {meas }}\left(\kappa ; \phi_{L 2}\right)$, best fit solutions for (b) $\left|f_{\text {sim }}(\kappa ; \phi)\right|^{2}$ and (c) transmission function $T(\kappa)$, and (d) final corrected spectra $\left|f_{\text {meas }}\left(\kappa ; \phi_{L 2}\right)\right|^{2}=$ $I_{\text {meas }}\left(\kappa ; \phi_{L 2}\right) / T(\kappa)$. 

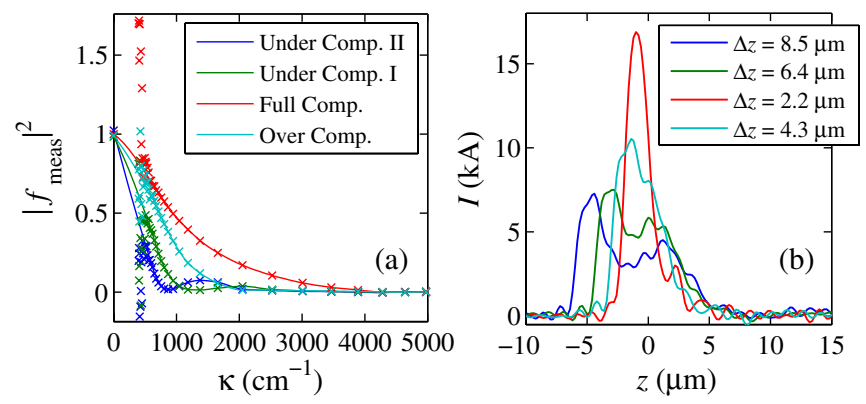

FIG. 5 (color online). Example beam profile reconstruction for $13.4 \mathrm{GeV}, 150 \mathrm{pC}$ electron bunches at four different $L 2 \mathrm{rf}$ phase settings around full compression showing (a) data and interpolated curves up to $\kappa=5000 \mathrm{~cm}^{-1}$ with (b) resulting current profiles and true FWHM bunch lengths $\Delta z$.

$150 \mathrm{pC}$ beam. Four examples are shown in Fig. 5: two of the under-compressed beam $\left(\left|\phi_{L 2}\right|<\left|\phi_{\text {full }}\right|\right)$, one at full compression, and one over compressed $\left(\left|\phi_{L 2}\right|>\left|\phi_{\text {full }}\right|\right)$. Spectrum curves are cubic interpolants of the measured points to provide over sampling for phase reconstruction. Because of weak transmission for $\kappa \lesssim 500 \mathrm{~cm}^{-1}$ (see Fig. 4), these points are discarded for interpolation. Relative scaling to the added point at $\kappa=0$ is known only from the amplitude calibration procedure and independent of bunch length. Using measured values, $T(\kappa)$ is scaled with the approximately logarithmic beam energy dependence of $I_{e}$ [8] and $N^{2}$ dependence of Eq. (3). Interpolants are used to retrieve $\phi_{\min }$ which is used to invert the Fourier transform, recovering $\rho(z)$.

The beam currents $I(z)=Q \rho(z)$ are shown in Fig. 5(b), with corresponding FWHM bunch lengths $\Delta z$. For both undercompressed cases, an expected double-horn structure appears in the currents showing small head and tail bumps [3].

Reconstruction was applied to the full chirp scan from which these examples were taken. A simulated scan using the set machine parameters was also performed. These are shown in Fig. 6 with simultaneous BLM2 measurements (Fig. 1). No fitting between simulation and measurement was used.

As BLM2 is calibrated assuming a square pulse, the values $\Delta z$ in Fig. 6 are from square-pulse fitting of all beam current profiles. The spectrometer agrees well with simulation with both having minima of $5.0 \mu \mathrm{m}$. Full compression phases at the spectrometer and BLM2 are $\phi_{\text {full }}=$ $-39.0^{\circ}$ and $-38.4^{\circ}$, respectively. Curves converge around $\phi_{L 2}=-36^{\circ}$, corresponding to the under compressed II case in Fig. 5. This is the typical set point for LCLS operations delivering minimum projected electron energy spread. The minimum $\Delta z$ of $17 \mu \mathrm{m}$ for BLM2 is believed to be due to a $30 \mu \mathrm{m}$ long-pass filter used to block optical wavelengths. The deviation of the spectrometer result for $\Delta z>18 \mu \mathrm{m}$ stems from the poor sensitivity for $\kappa<500 \mathrm{~cm}^{-1}(\lambda>20 \mu \mathrm{m})$, discussed below.

Performance is better in the shorter bunch regime. Pursuant to low-charge studies, measurement of 20 and
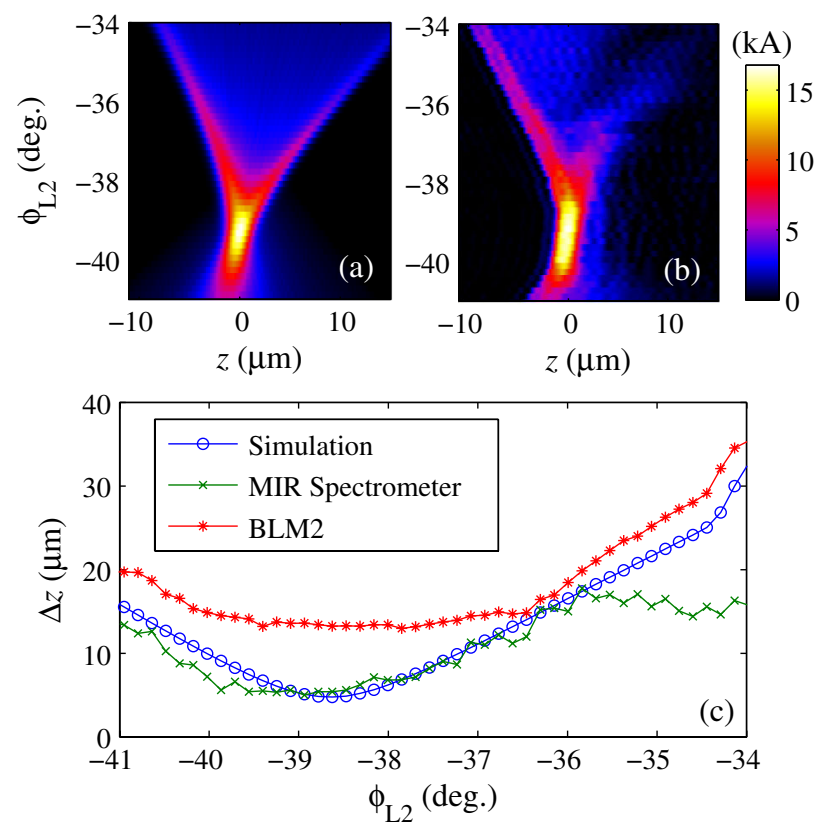

FIG. 6 (color online). Comparisons for the beam current profile dependence on $L 2 \mathrm{rf}$ phase for a $13.4 \mathrm{GeV}, 150 \mathrm{pC}$ electron bunch with (a) simulated profiles as a function of $\phi_{L 2}$, (b) profiles reconstructed from measured MIR spectra, and (c) FWHM bunch lengths $\Delta z$ from square current profile fitting including LCLS BLM2 measurement.

$10 \mathrm{pC}$ bunches yield minimum FWHM bunch lengths $\Delta z$ of 1.3 and $0.7 \mu \mathrm{m}$, respectively. In these cases, however, extrapolation of the high- $\kappa$ tail beyond the spectrometer $\kappa=9000 \mathrm{~cm}^{-1}$ cutoff is required. Furthermore, the signal levels approach the noise level of the detector array.

Improvements continue to push both long- and shortbunch boundaries. In the short-bunch limit a silicon filter window on the detector cuts off near-IR wavelengths to prevent photocurrent generation in the integrated circuit. A sensor without this requirement is being evaluated to both extend transmission below $1 \mu \mathrm{m}$ and double the intensity of light reaching the array making $<10 \mathrm{pC}$ bunch profile measurements possible. For longer bunches, zinc selenide neutral density filters were used to attenuate the CTR that saturates the array for $Q \gtrsim 40 \mathrm{pC}$. Though filter response has been measured to be flat over most of the spectrometer range [24], their absorption is strong for $\kappa<$ $500 \mathrm{~cm}^{-1}$. KRS-5 neutral density filters are being explored to correct this.

In summary, a novel, compact, MIR prism spectrometer has been designed, calibrated, and successfully demonstrated as a single-shot bunch length monitor for the LCLS. A combination beam- and simulation-based spectrometer characterization was demonstrated partially circumventing the need for an additional MIR source. Though not an ideal or independent solution, this approach naturally accounts for all spectral effects present in the system including difficult to quantify CTR imaging contributions. While demonstrated using CTR, noninterceptive processes 
such as coherent edge or diffraction radiation can be used to make it an online diagnostic.

Initial performance has been compared to independent measurement and simulation. Combining measured spectra with longitudinal profile reconstruction, bunch structure is resolved with reasonable agreement to simulation. Bunch lengths spanning $0.7-18 \mu \mathrm{m}$ FWHM over a range from 10-150 pC have been reported, exceeding design expectations in the low-charge, short-bunch regime and with improvements underway to better utilize the full bandwidth accessible to the spectrometer. Already integrated with LCLS, this MIR spectrometer continues to operate in support of FEL performance improvement studies.

We thank Axel Brachmann, Franz-Josef Decker, Sasha Gilevich, Rick Iverson, Gene Kraft, Jennifer Loos, Agostino Marinelli, Mike Minitti, Joe Robinson, and Jim Turner of SLAC National Accelerator Laboratory for their valuable contributions and support. This work was supported in part by U.S. Department of Energy Contract No. DE-AC02-76SF00515.

*tmaxwell@slac.stanford.edu

[1] E. Esarey, C. B. Schroeder, and W. P. Leemans, Rev. Mod. Phys. 81, 1229 (2009).

[2] P. Emma et al., Nat. Photonics 4, 641 (2010).

[3] Y. Ding et al., Phys. Rev. Lett. 102, 254801 (2009).

[4] Y. Ding, C. Behrens, P. Emma, J. Frisch, Z. Huang, H. Loos, P. Krejcik, and M.-H. Wang, Phys. Rev. ST Accel. Beams 14, 120701 (2011).

[5] P. Emma, J. Frisch, and P. Krejcik, SLAC Technical Report No. LCLS-TN-00-12, 2000.

[6] P. Krejcik et al., in Proceedings of the International Beam Instrumentation Conference, IBIC2012, Tsukuba, Japan, 2012, p. TUPA41.

[7] J.D. Jackson, Classical Electrodynamics (Wiley, New York, 1998), 3rd ed.

[8] V. L. Ginzburg and I. M. Frank, J. Phys. (USSR) 9, 353 (1945).

[9] S. Casalbuoni, B. Schmidt, P. Schmüser, V. Arsov, and S. Wesch, Phys. Rev. ST Accel. Beams 12, 030705 (2009).
[10] M. Castellano, A. Cianchi, G. Orlandi, and V. A. Verzilov, Nucl. Instrum. Methods Phys. Res., Sect. A 435, 297 (1999).

[11] A. G. Shkvarunets and R. B. Fiorito, Phys. Rev. ST Accel. Beams 11, 012801 (2008).

[12] H. Loos, T. Borden, P. Emma, J. Frisch, and J. Wu, in 2007 IEEE Particle Accelerator Conference (PAC) Albuquerque, NM, 2007 (IEEE, Piscataway, NJ, 2007), pp. 4189-4191.

[13] M. Dunning, G. Travish, A. Cohen, P. Frigola, S. Reiche, and J. Rosenzweig, Int. J. Mod. Phys. A 22, 4125 (2007).

[14] H.-c. Lihn, P. Kung, C. Settakorn, H. Wiedemann, and D. Bocek, Phys. Rev. E 53, 6413 (1996).

[15] A. Murokh, J. B. Rosenzweig, M. Hogan, H. Suk, G. Travish, and U. Happek, Nucl. Instrum. Methods Phys. Res., Sect. A 410, 452 (1998).

[16] D. Mihalcea, C. L. Bohn, U. Happek, and P. Piot, Phys. Rev. ST Accel. Beams 9, 082801 (2006).

[17] L. Frölich, Report No. DESY-THESIS 2005-11, 2005.

[18] J. Thangaraj et al., Rev. Sci. Instrum. 83, 043302 (2012).

[19] Y. Sugiyama, Y. Shibata, S. Sasaki, K. Ishi, T. Tsutaya, T. Osaka, M. Ikezawa, M. Oyamada, and Y. Kondo, Bull. Res. Inst. Sci. Meas., Tohoku Univ. 48, 1 (1999).

[20] T. Watanabe et al., Nucl. Instrum. Methods Phys. Res., Sect. A 480, 315 (2002).

[21] S. Wesch, B. Schmidt, C. Behrens, D.-H. Hossein, and P. Schmuüser, Nucl. Instrum. Methods Phys. Res., Sect. A 665, 40 (2011).

[22] T. Maxwell, Y. Ding, A. Fisher, J. Frisch, and H. Loos, in Proceedings of the International Beam Instrumentation Conference, IBIC2012, Tsukuba, Japan, 2012, p. TUPA47.

[23] W. S. Rodney and I. H. Maliston, J. Opt. Soc. Am. 46, 956 (1956).

[24] M. Martin, Advanced Light Source, Lawrence Berkeley National Laboratory, Berkeley, CA, (private communication).

[25] K. Bane and P. Emma, in 2005 IEEE Particle Accelerator Conference (PAC): Knoxville, TN, 2005 (IEEE, Piscataway, NJ, 2005), pp. 4266-4268.

[26] J. S. Toll, Phys. Rev. 104, 1760 (1956).

[27] R. Lai and A. J. Sievers, Phys. Rev. E 50, R3342 (1994).

[28] R. Lai and A. Sievers, Nucl. Instrum. Methods Phys. Res., Sect. A 397, 221 (1997). 